\title{
Holmium Nanoparticles: Preparation and In Vitro Characterization of a New Device for Radioablation of Solid Malignancies
}

\author{
Wouter Bult • Rosanne Varkevisser • Fouad Soulimani • Peter R. Seevinck • Hendrik de Leeuw • Chris J. G. Bakker • Peter R. Luïten • \\ Alfred D. van het Schip • Wim E. Hennink • J. Frank W. Nijsen
}

Received: 12 April 2010 / Accepted: 19 July 2010 / Published online: 31 July 2010

(C) The Author(s) 2010. This article is published with open access at Springerlink.com

\begin{abstract}
Purpose The present study introduces the preparation and in vitro characterization of a nanoparticle device comprising holmium acetylacetonate for radioablation of unresectable solid malignancies.
\end{abstract}

Methods HoAcAc nanoparticles were prepared by dissolving holmium acetylacetonate in chloroform, followed by emulsification in an aqueous solution of a surfactant and evaporation of

\author{
W. Bult • R. Varkevisser • P. R. Luijten • A. D. van het Schip • \\ J. F. W. Nijsen $(\square)$ \\ Department of Radiology and Nuclear Medicine, Imaging Division \\ University Medical Centre Utrecht \\ Heidelberglaan 100 \\ 3584 CX Utrecht, The Netherlands \\ e-mail: f.nijsen@umcutrecht.n! \\ W. Bult $\cdot$ W. E. Hennink \\ Department of Pharmaceutics \\ Utrecht Institute for Pharmaceutical Sciences, Utrecht University \\ Sorbonnelaan 16. P.O. Box 80082
}

3508 TB Utrecht, The Netherlands

\section{F. Soulimani}

Inorganic Chemistry and Catalysis Group, Department of Chemistry Faculty of Science, Utrecht University

P.O. Box 80083, 3508 TB Utrecht, The Netherlands

P. R. Seevinck • H. de Leeuw • C. J. G. Bakker

Imaging Sciences Institute, Imaging Division

University Medical Centre Utrecht

Heidelberglaan 100

3584 CX Utrecht, The Netherlands

Present Address:

R. Varkevisser

Department of Medical Physiology, Division Heart and Lungs

University Medical Centre Utrecht

Heidelberglaan 100

3584 CX Utrecht, The Netherlands the solvent. The diameter, surface morphology, holmium content, and zeta potential were measured, and thermal behavior of the resulting particles was investigated. The stability of the particles was tested in HEPES buffer. The $r_{2}{ }^{*}$ relaxivity of protons and mass attenuation coefficient of the nanoparticles were determined. The particle diameter and surface morphology were studied after neutron activation.

Results Spherical particles with a smooth surface and diameter of $78 \pm 10 \mathrm{~nm}$ were obtained, and the particles were stable in buffer. Neutron irradiation did not damage the particles, and adequate amounts of activity were produced for nuclear imaging and radioablation of malignancies through intratumoral injections.

Conclusions The present study demonstrates that HoAcAc nanoparticles were prepared using a solvent evaporation process. The particle diameter can easily be adapted and can be optimized for specific therapeutic applications and tumor types.

KEY WORDS ablation · characterization · holmium . nanoparticles · tumor

\section{INTRODUCTION}

Solid tumors represent the majority of all diagnosed cancer cases in 2009, accounting for more than 1.3 million new cases in the USA and approximately $90 \%$ of the cancerrelated deaths (1). Treatment options of solid tumors with a limited number of metastases include surgical resection, external beam radiotherapy, chemotherapy, and locoregional ablation. Surgery is the preferred treatment option, since this can be regarded as a curative treatment (2). For patients not amenable for surgical resection, external beam radiotherapy is often used curatively. However, due to radiation sensitivity of the adjacent tissue or motion 
artifacts, not all tumors can be considered for this therapy. In most cases, chemotherapy is applied as palliative treatment. Percutaneous locoregional thermoablative techniques (e.g. radiofrequency ablation and high intensity focused ultrasound) are increasingly applied with promising results in a number of different tumor types, including tumors in the liver, lungs, and kidneys $(2,3)$. One of the drawbacks associated with these thermoablative therapies is the transport of heat from the tumors by blood vessels, leading to incomplete tumor eradication (3).

The intratumoral administration of beta-emitting radionuclides is an alternative to overcome the drawbacks associated with thermoablative techniques, whilst retaining the specificity of local ablation. The intratumoral administration of high energy beta-emitting microspheres has been successfully tested in patients suffering from liver tumors. Yttrium-90-loaded glass microspheres showed a significant tumor response in 24 of 27 patients (4), whereas phosphorous-32-containing nanocolloids (5) showed a significant tumor response in 12 of 17 patients. However, direct visualization of beta particles with a gamma camera is not possible, making these isotopes unsuitable for accurate dosimetric calculations. These drawbacks can be overcome by intratumoral administration of combined beta and gamma emitters. Holmium-166 $\left({ }^{166} \mathrm{Ho}\right)$ is a very attractive candidate, since this radionuclide emits gamma rays in addition to high-energy beta particles, allowing for both nuclear imaging and radioablation, respectively. Moreover, holmium can be visualized by CT and MRI due to its high attenuation coefficient and paramagnetic properties (6). The intratumoral injection of ${ }^{166} \mathrm{Ho}$ nitrate $\left({ }^{166} \mathrm{Ho}\left(\mathrm{NO}_{3}\right)_{3}\right)$ has shown promising results in a rat model of malignant melanoma. A single injection of holmium nitrate distributed throughout the tumor, leading to a significant tumor size reduction (7). A clinically applied ${ }^{166} \mathrm{Ho}\left(\mathrm{NO}_{3}\right)_{3}$-loaded device for intratumoral injections is ${ }^{166} \mathrm{Ho}\left(\mathrm{NO}_{3}\right)_{3}$-labelled chitosan (8). Response rates were high in patients with hepatocellular carcinoma $(31 / 40)$, but due to leakage of ${ }^{166} \mathrm{Ho}$ to the systemic circulation in more than $25 \%$ of patients $(11 / 40)$, hematopoietic suppression was observed. Microspheres with an ultra high holmium content for intratumoral administration were developed to overcome these side effects (9) and were successfully evaluated in a murine renal cell carcinoma model (10). Tumor growth was arrested, and no holmium was observed outside the tumor on SPECT, GT and MRI. Although the treatment showed promising results, only half of the intended dose was administered, due to premature settling of particles in the syringe and the formation of aggregates. After administration, the microspheres remained at the site of injection due to their size.

The aim of this study is to develop and characterize a holmium-containing device that takes advantage of the favorable properties of micro-sized particles, and circumvents their aforementioned downsides. In this paper, we present the development and characterization of nano-sized holmium acetylacetonate particles as a potential radioablation device for solid malignancies.

\section{MATERIALS AND METHODS}

All chemicals were commercially available and used as obtained. Acetylacetone, 2,4-pentanedione (AcAc; >99.9\%), chloroform (HPLC-grade), didodecyldimethylammonium bromide (DMAB; >98\%), 4-(2-Hydroxyethyl)piperazine-1ethanesulfonic acid (HEPES; >99\%), polyvinyl alcohol (PVA, average MW 30,000-70,000) and sodium hydroxide $(\mathrm{NaOH} ; 99 \%)$ were purchased from Sigma Aldrich (Steinheim, Germany). Seakem LE Agarose was purchased from Lonza (Basel, Switzerland). Holmium (III) chloride hexahydrate $\left(\mathrm{HoCl}_{3} .6 \mathrm{H}_{2} \mathrm{O} ; 99.95 \%\right)$ was purchased from Metall Rare Earth Ltd. (Shenzhen, China).

\section{Particle Preparation}

Holmium acetylacetonate was prepared as described earlier (11). HoAcAc nanoparticles were prepared by solvent evaporation after emulsification. HoAcAc crystals were dissolved in chloroform $(5.5 \%(w / w))$ and subsequently recrystallized by rapid evaporation of the chloroform under a continuous flow of nitrogen. The recrystallized HoAcAc was dissolved in chloroform $(5.5 \%,(w / w))$, which was emulsified in an aqueous phase containing a stabilizer (PVA or DMAB, both $2 \% \mathrm{w} / \mathrm{v}$ ) using an Ultra-turrax homogenizer (Ultra-turrax T25 basic, IKA, Staufen, Germany) at a stirrer speed of 9,500; 13,500; 20,500; or $24,000 \mathrm{rpm}$ for $5 \mathrm{~min}$. After emulsification, the organic solvent was evaporated using a rotator evaporator, and the nanoparticles were collected by centrifugation $(25,000 \mathrm{rpm}$ for $25 \mathrm{~min}$ at $4^{\circ} \mathrm{C}$ ) and washed 6 times with water. The particles were lyophilized overnight using a Christ "Alpha $1-2$ " freeze dryer.

\section{Particle Characterization}

Nanoparticle diameter was measured by dynamic light scattering (DLS) using a Malvern ALV GGS-3 (Malvern Instruments, Malvern, UK) equipped with a JDS Uniphase $22 \mathrm{~mW} \mathrm{He}-\mathrm{Ne}$ laser operating at $632.8 \mathrm{~nm}$ at a $90^{\circ}$ angle. Autocorrelation functions were analyzed by the cumulants method by fitting a single exponential to the correlation function to obtain the mean size and the PDI, using the ALV-60 $\times 0$ software V.3.X provided by Malvern. DLS results were recorded as the number weighted mean diameter and Z-average diameter, both in $\mathrm{nm}$. 
Scanning electron microscopy (SEM) was performed on samples that were mounted on an aluminum stub and coated with a $6 \mathrm{~nm}$ layer of platinum. SEM micrographs were collected on a FEI FEG-SEM XL30 (FEI Company, Eindhoven, The Netherlands) at a voltage of $5 \mathrm{kV}$.

Elemental analysis of carbon, hydrogen, oxygen, bromine and holmium of HoAcAc nanoparticles, HoAcAc crystals, and recrystallized HoAcAc after evaporation of chloroform was performed by $\mathrm{H}$. Kolbe, Mülheim an der Ruhr, Germany.

Fourier Transform Infrared Spectroscopy of freeze-dried HoAcAc nanoparticles, holmium acetylacetonate crystals, and recrystallized crystals was performed using a Perkin Elmer SpectrumOne FT-IR spectrometer (PerkinElmer, Groningen, The Netherlands). The spectra were recorded using $\mathrm{KBr}$ pellets by accumulating 256 scans per spectrum.

A DSC Q1000 (TA instruments Inc., Etten-Leur, The Netherlands) was used for performing modulated differential scanning calorimetry. Scans were recorded from 25$220^{\circ} \mathrm{C}$ at a heating rate of $2^{\circ} \mathrm{Cmin}^{-1}$ and a modulation temperature of $1^{\circ} \mathrm{Cmin}^{-1}$.

Zeta potentials of the HoAcAc nanoparticles were measured using a Zetasizer Nano (Malvern Instruments, Malvern, UK). Samples of lyophilized nanoparticles were suspended in $10 \mathrm{mM}$ HEPES at $\mathrm{pH} 7.4$ at a concentration of $5 \mathrm{mg} / \mathrm{ml}$ and sonicated for $10 \mathrm{~min}$; thereafter, the zeta potential was measured. Reported values are the mean of 3 measurements.

The stability of the HoAcAc particles prepared using DMAB and PVA was studied by dispersing them in a HEPES buffer at $\mathrm{pH} 7.4(10 \mathrm{mM})$. At different time points $(t=0,1,2$, 4, 8, 24, 48 and $72 \mathrm{~h}$ ), the particle diameter was measured using DLS at $25^{\circ} \mathrm{C}$, as described before. The release of holmium from the HoAcAc particles was determined by transferring $1 \mathrm{ml}$ of a $50 \mathrm{mg} / \mathrm{ml}$ suspension of HoAcAc nanoparticles (either prepared in PVA or DMAB) in $10 \mathrm{mM}$ HEPES ( $\mathrm{pH}$ 7.4) to a Slide-a-Lyzer Dialysis Cassette (Thermo Scientific, Etten-Leur, The Netherlands) with a 10,000 MWCO membrane. All release samples were prepared in triplicate. The cassettes were dialyzed against $500 \mathrm{ml}$ HEPES buffer (10 mM, pH 7.4). At different timepoints $(t=0,1,2,4,8,24,48$ and $72 \mathrm{~h}), 1 \mathrm{ml}$ of sample was taken. Four $\mathrm{ml} \mathrm{HNO}_{3} 5 \%(v / v)$ were added, and the holmium content of the resulting solutions was measured using inductively coupled plasma optical emission spectroscopy (ICP OES). Samples were measured at three different wavelengths $(339.898,345.600$ and $347.426 \mathrm{~nm})$ in an Optima 4300 CV (PerkinElmer; Norwalk, USA).

\section{Multimodality Imaging}

To determine the sensitivity of MRI and X-ray CT for the HoAcAc nanoparticles, phantom experiments were carried out on MRI and CT. The phantom used to determine MRI and CT sensitivity consisted of agarose $(2 \%(w / w))$ gel samples in which HoAcAc nanoparticles were homogeneously dispersed (loadings ranged from 0 to $8 \mathrm{mg}$ nanoparticles per $\mathrm{ml}$ agarose) in glass tubes (diameter $10 \mathrm{~mm}$, length $75 \mathrm{~mm}$ ). Sample tubes were positioned parallel to the $\mathrm{B}_{0}$ field in the MR scanner. MR imaging was performed using a 1.5 and a 3 Tesla clinical MR scanner (Intera, Achieva, respectively, Philips Healthcare, Best, The Netherlands). The sensitivity of gradient echo MRI for a paramagnetic contrast agent can be expressed in terms of its $\mathrm{r}_{2} *$ relaxivity (in $\left[\mathrm{sec}^{-1} \mathrm{mg}^{-1} \mathrm{ml}\right.$ ), which represents the change in the effective proton transverse relaxation rate ( $\mathrm{R} 2 *$ in $\left[\mathrm{sec}^{-1}\right]$ ) per unit concentration (in $\mathrm{mg} / \mathrm{ml}$ ). The $\mathrm{R} 2 *$ relaxation rate characterizes the rate of monoexponential signal decay due to transverse relaxation. To determine the $\mathrm{r}_{2} *$ relaxivity of HoAcAc particles, the R2* relaxation rates of the HoAcAc dilution series were determined with a multiple gradient echo MR sequence, acquiring 15 echos with an echo spacing of $1.39 \mathrm{msec}$, a repetition time of $500 \mathrm{msec}$, a field of view (FOV) of $128 \times$ $128 \times 20 \mathrm{~mm}$, a voxel size of $1 \times 1 \times 5 \mathrm{~mm}$, two signal averages and a $60^{\circ}$ flip angle. Linear regression of the R2* values and the HoAcAc concentrations provided the $\mathrm{r}_{2}$ * relaxivity (6). For qualitative purposes, a second agarose phantom was constructed, with depots of HoAcAc nanoparticles in decreasing concentrations by serial dilution (ranging from $100 \mu \mathrm{g}$ to $1.5 \mu \mathrm{g}$ nanoparticles). To obtain cavities of a reproducible size in this gel, $2 \mathrm{ml}$ pipette tips were positioned in the gel prior to setting of the gel. After setting of the gel, the pipette tips were removed, and $50 \mu \mathrm{l}$ of a homogeneous HoAcAc nanoparticle suspension was pipetted into the cavities thus obtained. The particles were left to settle, followed by filling with agarose gel. This phantom was positioned coaxially in a 3 Tesla clinical MR scanner and used to determine the minimal amount of HoAcAc particles that can be visualized using MR imaging. Imaging parameters were similar for this phantom setup, apart from the $15^{\circ}$ flip angle.

Quantitative X-ray CT imaging was performed on a clinical 64 slice CT scanner (Brilliance, Philips Medical Systems, Best, The Netherlands). The sample tubes were positioned co-axially to the main axis of the scanner. Using the methods described by Seevinck et al., a linear regression curve was constructed to determine the sensitivity of X-ray CT for HoAcAc nanoparticles (6).

Neutron irradiations were performed in the pneumatic rabbit system operational in a nuclear research reactor (Department of Radiation, Radionuclides and Reactors, Delft University of Technology, Delft, The Netherlands) (12). Approximately $50 \mathrm{mg}$ HoAcAc nanoparticles were accurately weighed and transferred into a high-density polyethylene vial (Posthumus plastics, Beverwijk, the Netherlands) and 
irradiated for 30 or $60 \mathrm{~min}$ in a facility with a thermal neutron flux of $5 \times 10^{12} \mathrm{n} \mathrm{cm}^{-2} \mathrm{~s}^{-1}$. After neutron irradiation, the radioactivity was measured. To ensure safe handling of the nanoparticles for SEM image acquisition and DLS measurements, the ${ }^{166} \mathrm{HoAcAc}$ nanoparticles were left to decay for 1 month.

\section{RESULTS AND DISCUSSION}

HoAcAc nanoparticles were prepared using solvent evaporation technology. In short, recrystallized HoAcAc was dissolved in chloroform, and the resulting solution was emulsified in an aqueous solution with $2 \%(w / w)$ PVA followed by evaporation of the solvent. Particles with a number weighted mean diameter ranging from $1,708 \pm 276$ to $799 \pm 147 \mathrm{~nm}$ were obtained when increasing the stirrer speed from 9,500 to 24,000 rpm (see Table I and Fig. 1). The yield for all batches was $51 \pm 2 \%$. Scanning electron microscopy (SEM) revealed spherical particles with a rugged surface (Fig. 1a), with a similar particle diameter as the number weighted particle diameter observed with dynamic light scattering (DLS). A larger z-average particle diameter was measured owing to the presence of a few large particles (13) (Table I).

To decrease the particle diameter, the cationic surfactant didodecyldimethylammonium bromide (DMAB) was used as an emulsifier (14). With increasing stirrer speed from 9,500 to $24,000 \mathrm{rpm}$, the number weighted mean particle diameter substantially decreased from $1,168 \pm 252$ to $78 \pm$ $10 \mathrm{~nm}$ (Table I). The yield for all batches was $47 \pm 5 \%$. Spherical particles with a smooth surface were observed with SEM (Fig. 1b). The smaller diameter of the particles using DMAB instead of PVA can be explained by the lower critical micelle concentration for DMAB compared to PVA (14). Consequently, DMAB more readily stabilizes chloroform droplets in emulsion, promoting the formation of small chloroform droplets. Particle characterization was performed on the particles with a mean diameter of around $78 \mathrm{~nm}$ prepared with DMAB at a stirrer speed of
24,000 rpm. When PVA particles were used as a comparison, the particles prepared at 24,000 rpm in PVA were used.

Table II shows that the holmium content of the HoAcAc nanoparticles, as determined by elemental analysis, was $43.7 \%(w / w)$, which is higher than the holmium content of the HoAcAc crystals $(31.9 \%(w / w))$ used as the starting material. An increase in holmium content was also observed in a previous study on the preparation of HoAcAc microspheres, which was attributed to a loss of acetylacetonate during reorganization of acetylacetonate around Ho (III) ions during the gradual evaporation of the solvent (9). Interestingly, HoAcAc crystals also showed an increase in holmium content from $31.9 \%(w / w)$ to $38.3 \%(w / w)$ after dissolution of the HoAcAc crystals in chloroform followed by recrystallization. The holmium content in the HoAcAc nanoparticles was further increased to $43.7 \%$. The bromine content was also determined in the HoAcAc nanoparticles prepared in DMAB, to determine the amount of emulsifier present after washing. The amount of bromine present was $0.8 \%(w / w)$, after washing 6 times with water, which is representative of $0.8 \%$ of DMAB. Infrared spectroscopy, elemental analysis and differential scanning calorimetry (DSC) were performed to investigate the HoAcAc nanoparticles and the HoAcAc crystals. The infrared spectra of the original HoAcAc crystals, recrystallized HoAcAc and the HoAcAc nanoparticles, irrespective of the preparation characteristics, show characteristic peaks for acetylacetonate at 1,517 and $1,610 \mathrm{~cm}^{-1}$ representing the $\mathrm{C}=\mathrm{C}$ and the $\mathrm{C}=\mathrm{O}$ stretching bands of acetylacetonate, respectively (15) (Fig. 2). This is a confirmation that acetylacetonate is present in both the recrystallized HoAcAc and the nanoparticles. The presence of small but sharp peaks in the blue spectrum (HoAcAc crystals) at around $3,600 \mathrm{~cm}^{-1}$ is an indication that crystal water is present in the HoAcAc crystals (16). These peaks are less pronounced in the black and red spectrum, indicating that the structure of HoAcAc crystals has changed during particle preparation and water was lost from the complex. The more pronounced peaks in the red spectrum (HoAcAc nanoparticles) around 2,800-3,000 $\mathrm{cm}^{-1}$
Table I Effects of Preparation Variables on the Particle Size and Polydispersity Index $(n=3)$ of HoAcAc Nanoparticles

${ }^{a}$ results displayed as particle diameter \pm standard deviation in $\mathrm{nm}$

PDI Polydispersity Index: a measure to determine the heterogeneity of the particle size, I being completely heterodisperse, 0 being completely monodisperse.

\begin{tabular}{llrrr}
\hline Stirrer speed (rpm) & Emulsifier & $\begin{array}{c}\text { Particle diameter } \\
\text { Number weighted mean }\end{array}$ & $\begin{array}{l}\text { Particle diameter } \\
\text { Z-average }^{a}\end{array}$ & PDI \\
\hline 9,500 & $2 \%$ PVA & $1,708 \pm 276$ & $2,143 \pm 687$ & 0.26 \\
13,500 & $2 \%$ PVA & $1,153 \pm 259$ & $1,685 \pm 29$ & 0.28 \\
20,500 & $2 \%$ PVA & $1,002 \pm 161$ & $1,414 \pm 128$ & 0.24 \\
24,000 & $2 \%$ PVA & $799 \pm 147$ & $1,175 \pm 149$ & 0.25 \\
9,500 & $2 \%$ DMAB & $1,168 \pm 252$ & $1,677 \pm 789$ & 0.25 \\
13,500 & $2 \%$ DMAB & $653 \pm 166$ & $997 \pm 509$ & 0.27 \\
20,500 & $2 \%$ DMAB & $311 \pm 123$ & $659 \pm 68$ & 0.35 \\
24,000 & $2 \%$ DMAB & $78 \pm 10$ & $462 \pm 265$ & 0.21 \\
\hline
\end{tabular}



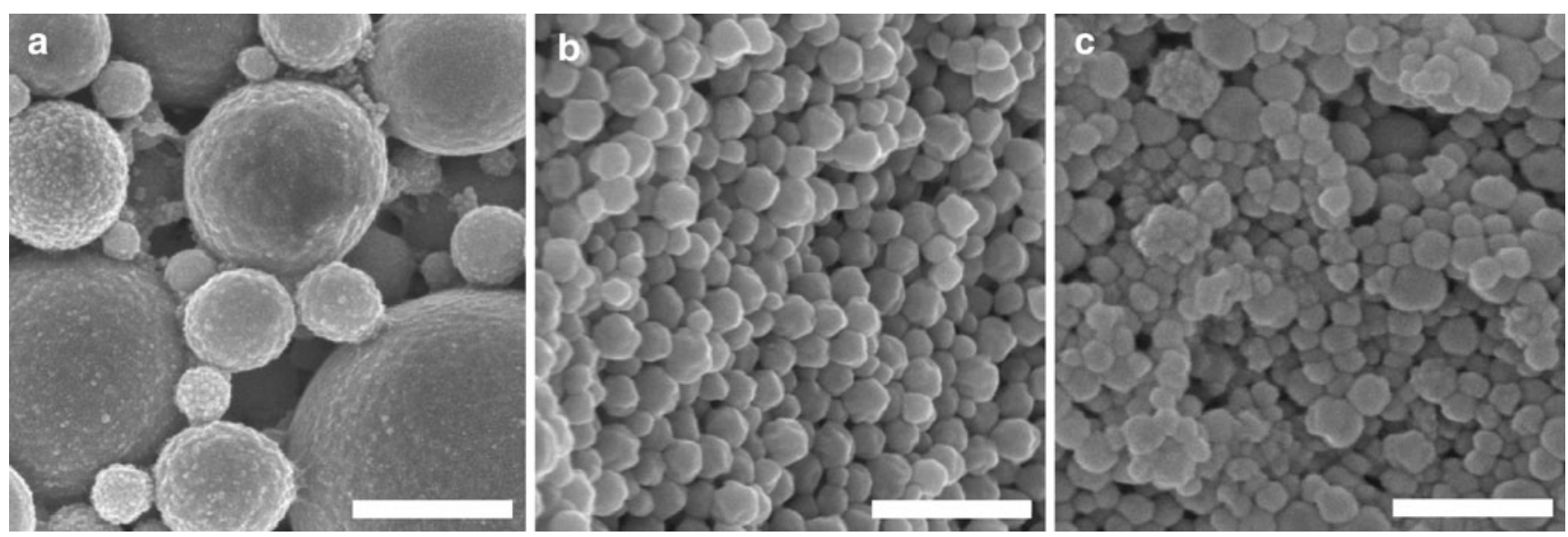

Fig. I Scanning electron micrographs of HoAcAc nanoparticles showing the effect of emulsifier on particle diameter and surface a particles prepared using PVA as emulsifier, b particles prepared using DMAB as emulsifier (stirrer speed was 24,000 rpm for both formulations), c particles prepared using DMAB as emulsifier after neutron irradiation ( $1 \mathrm{~h}$ at a neutron flux of $5 \times 10^{12} \mathrm{n} \mathrm{cm}^{-2} \mathrm{~s}^{-1}$ ). The magnification was 100,000x for all samples; bar represents $500 \mathrm{~nm}$.

can be explained by the presence of surfactant in the sample. These peaks are characteristic for aliphatic alkyl chains originating from $\mathrm{DMAB}$, the presence of which was determined by elemental analysis.

Based on results from elemental analysis in combination with the finding from IR spectroscopy that all forms contain acetylacetonate, HoAcAc crystals consist of three acetylacetonate ligands and three water molecules complexed with the $\mathrm{Ho}(\mathrm{III})$ ion, as reported previously (16). Elemental analysis of recrystallized HoAcAc gives evidence that 2.5 acetylacetonate molecules and one water molecule surround each $\mathrm{Ho}$ (III) ion, while in HoAcAc nanoparticles each $\mathrm{Ho}(\mathrm{III})$ is expected to be surrounded by two acetylacetonate molecules and one water molecule (Fig. 3, Table II).

Differential scanning calorimetry of HoAcAc crystals showed an endotherm at $126 \pm 11^{\circ} \mathrm{C}$ (Fig. 4a, Table III), which can be attributed to loss of water from the complex (15). Interestingly, the thermograms of recrystallized HoAcAc show endotherms at $126^{\circ} \mathrm{C}$ and $156^{\circ} \mathrm{C}$ (Fig. 4b, Table III), indicating the presence of a reorganized
HoAcAc structure after recrystallization from chloroform (Fig. 3). The HoAcAc nanoparticles showed an endotherm at $198 \pm 3^{\circ} \mathrm{C}$ (Fig. 4c, Table III), likely associated with a loss of water. The higher temperature needed to expel one water molecule is an indication that the HoAcAc in the nanoparticles is reorganized in a network-like structure. A schematic representation of the proposed reorganization process is shown in Fig. 3. Definite clarification of the structural rearrangement requires extensive further research.

The use of recrystallized HoAcAc was a prerequisite for the formation of nanoparticles. When HoAcAc crystals were used, small needle-like structures were formed instead of nanoparticles. In HoAcAc crystals, three acetylacetonate molecules are complexed with one $\mathrm{Ho}$ (III) ion. After recrystallization of HoAcAc, the $\mathrm{Ho}$ (III) ion loses half an acetylacetonate molecule, resulting in one acetylacetonate molecule that bridges between two $\mathrm{Ho}$ (III) ions. When dissolving the recrystallized HoAcAc in chloroform, followed by rapid evaporation of the chloroform, another half acetylacetonate is lost per Ho(III) ion. We expect this to lead to the formation of another acetylacetonate bridge between

Table II Elemental Analysis of HoAcAc Crystals, Recrystallized HoAcAc and HoAcAc Nanoparticles

\begin{tabular}{|c|c|c|c|c|c|c|}
\hline & \multicolumn{2}{|c|}{$\mathrm{HoAcAc}$ crystals $\left(\mathrm{HoAcAc}_{3} * 3 \mathrm{H}_{2} \mathrm{O}\right)$} & \multicolumn{2}{|c|}{ HoAcAc recrystallized $\left(\mathrm{Ho}_{2} \mathrm{AcAc}_{5} * 2 \mathrm{H}_{2} \mathrm{O}\right)$} & \multicolumn{2}{|c|}{ HoAcAc nanoparticles $\left(\mathrm{Ho}_{2} \mathrm{AcAc}_{4} * \mathrm{H}_{2} \mathrm{O}\right)$} \\
\hline & Calculated (\%) & Measured $^{a}(\%)$ & Proposed (\%) & Measured (\%) & Proposed (\%) & Measured (\%) \\
\hline C & 34.9 & 35.6 & 34.9 & 34.1 & 31.5 & 31.6 \\
\hline $\mathrm{H}$ & 5.3 & 5.3 & 4.6 & 4.4 & 4.2 & 4.4 \\
\hline$\bigcirc$ & 27.9 & 26.3 & 22.3 & 21.8 & 21.0 & 19.9 \\
\hline $\mathrm{Ho}$ & 31.9 & 32.6 & 38.3 & 37.6 & 43.3 & 43.7 \\
\hline $\mathrm{Br}$ & - & - & - & - & $<1 \%$ & 0.8 \\
\hline
\end{tabular}

a Measured values are presented as the mean of duplicate analyses. 


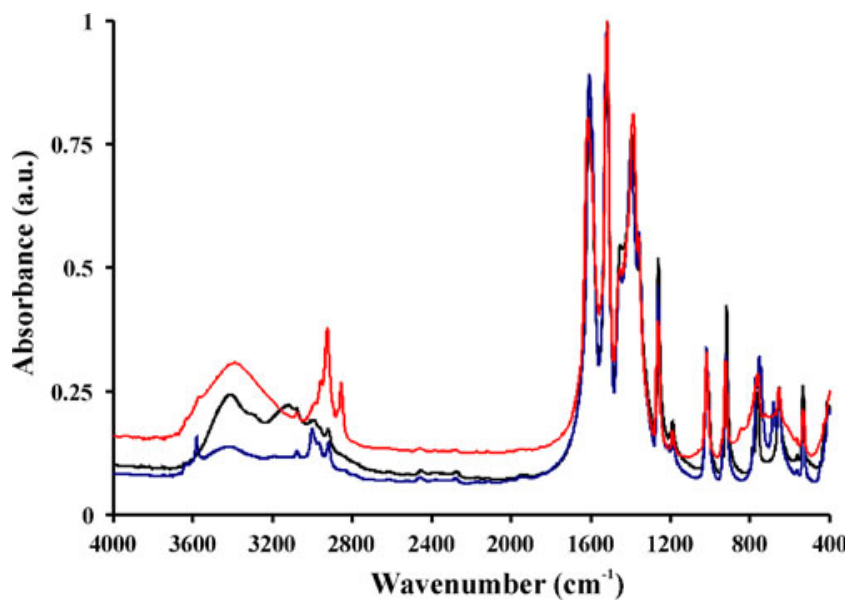

Fig. 2 IR absorbance spectra of HoAcAc crystals (in blue), recrystallized HoAcAc (in black) and HoAcAc nanoparticles prepared at 24,000 rpm in $D M A B$ (in red). The spectra were normalized to the peak at $1,517 \mathrm{~cm}^{-1}$.

two Ho(III) ions. The gradual removal of solvent is imperative in this particle preparation method, allowing acetylacetonate to exert a bridging effect between $\mathrm{Ho}(\mathrm{III})$ ions (17).

A positive zeta potential of $26.1 \pm 5.4 \mathrm{mV}$ was recorded for nanoparticles prepared with the cationic surfactant DMAB. The use of PVA as the stabilizer resulted in particles with a slightly positive zeta potential value of $3.4 \pm$ $5.0 \mathrm{mV}$. The stability of the particles was investigated by measuring the particle diameter over time. Particles prepared with DMAB as emulsifier did not show aggrega-

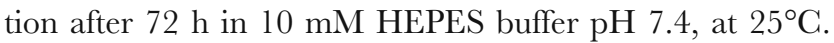
The positive zeta potential of particles prepared in DMAB creates repulsive forces between the particles, thereby preventing particle aggregation (18). Obviously, the zeta potential of particles prepared in PVA is insufficient to create these repulsive forces, leading to particle aggregation. Additionally, the stability of the HoAcAc nanoparticles was investigated by measuring the release of holmium from the particles after suspension for $72 \mathrm{~h}$ in $10 \mathrm{mM}$ HEPES buffer $\mathrm{pH} 7.4$ at $25^{\circ} \mathrm{C}$. The release of holmium from the nanoparticles prepared in DMAB was $1.9 \pm 0.1 \%$ after $72 \mathrm{~h}$. The release from the particles prepared in PVA was $7.6 \pm 4.7 \%$ after $72 \mathrm{~h}$. The particles

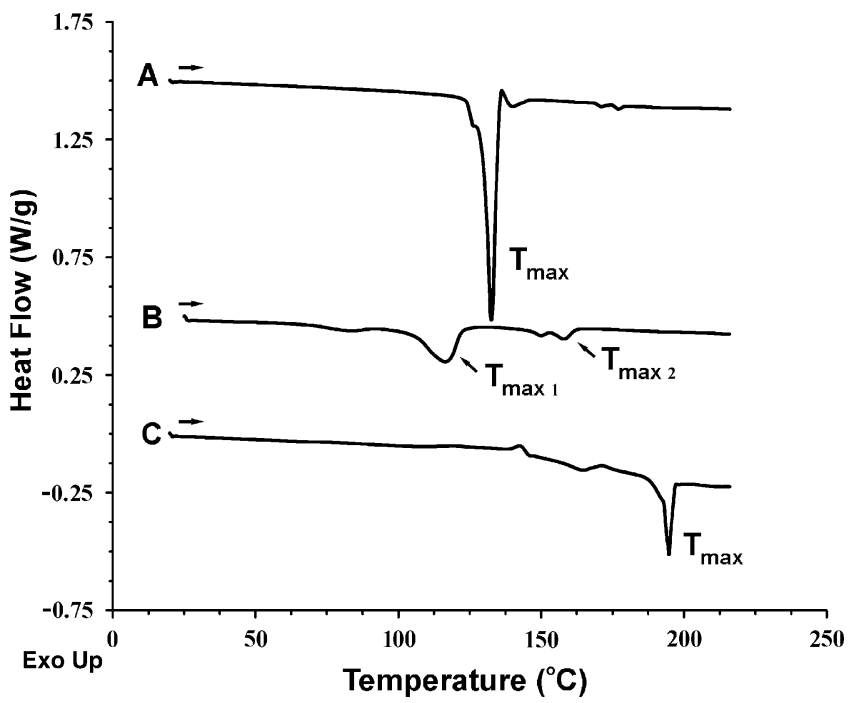

Fig. 4 mDSC thermograms of (A) HoAcAc crystals, (B) recrystallized HoAcAc and (C) HoAcAc nanoparticles. $T_{\max }$ is the peak maximum of the endotherm.

prepared in DMAB are more stable and thus seem more suitable for intratumoral radioablation. However, mid- and long-term stability experiments in different media (i.e. serum, lactates, citrates) are required prior to in vivo efficacy experiments.

The MR imaging characteristics were assessed on clinical 1.5 and 3 Tesla MR systems using agarose phantoms with manganese to mimic the susceptibility of liver tissue (19). The paramagnetic nature of holmium causes an enhanced transverse relaxation rate, which results in signal loss seen as blackening on $\mathrm{T}_{2} *$ weighted images (20). The MRI sensitivity of HoAcAc nanoparticles is described by the $r_{2}{ }^{*}$ and was calculated from transverse images using the method described by Seevinck et al. (6). The $\mathrm{r}_{2} *$ is $110 \mathrm{~s}^{-1} \mathrm{mg}^{-1} \mathrm{ml}$ (1.5 Tesla) and $295 \mathrm{~s}^{-1} \mathrm{mg}^{-1} \mathrm{ml}$ (3 Tesla), which was lower than expected, when compared to HoAcAc microspheres $\left(15 \mu \mathrm{m} ; \mathrm{r}_{2} * 264 \mathrm{~s}^{-1} \mathrm{mg}^{-1} \mathrm{ml}\right.$ at 1.5 Tesla (9)) with a similar holmium content $(45 \%$ w/w). This lower $\mathrm{r}_{2} *$ is likely caused by the particle size of the HoAcAc nanoparticles. The dephasing of spins for the HoAcAc microspheres of around $15 \mu \mathrm{m}$ follows a static regimen
Fig. 3 Schematic representation of the proposed different forms of HoAcAc complexes. In each subsequent step, acetylacetonate and one water molecule is lost. The remaining acetylacetonate molecules act as bridges between $\mathrm{Ho}(\mathrm{III})$ ions. (AcAc and $R=$ acetylacetonate)

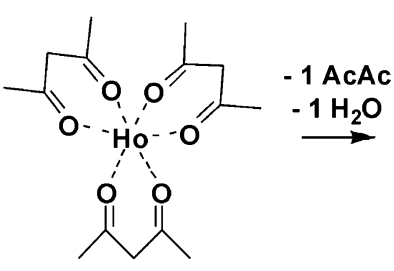

HoAcAc crystals $\mathrm{Ho}\left(\mathrm{C}_{5} \mathrm{H}_{7} \mathrm{O}_{2}\right)_{3}{ }^{*} 3 \mathrm{H}_{2} \mathrm{O}$
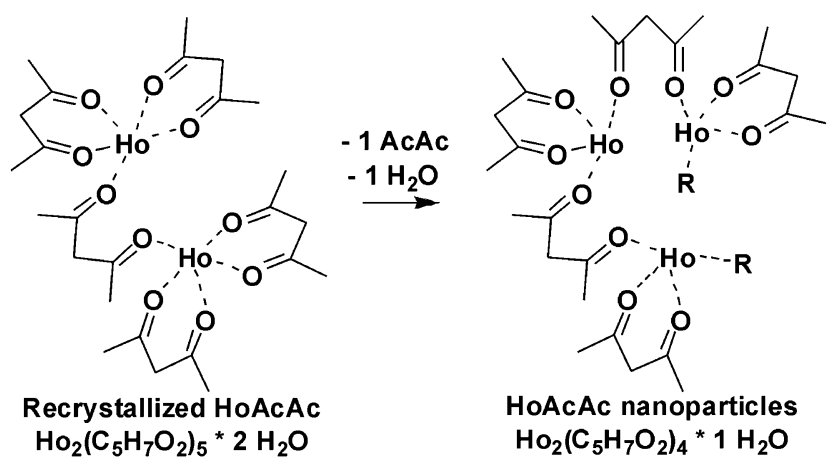
Table III Summary of DSC Results from HoAcAc Crystals, Before and After Recrystallization, and HoAcAc Nanoparticles $(n=4-6)$

\begin{tabular}{lrrr}
\hline & Onset $T_{\max }\left({ }^{\circ} \mathrm{C}\right)$ & $T_{\max }\left({ }^{\circ} \mathrm{C}\right)$ & \multicolumn{1}{c}{$\Delta H(\mathrm{~J} / \mathrm{g})$} \\
\hline HoAcAc crystals & $120 \pm 17$ & $126 \pm 11$ & $113 \pm 12$ \\
HoAcAc recrystallized & $98 \pm 12$ & $112 \pm 8$ & $43 \pm 9$ \\
& $147 \pm 9$ & $154 \pm 7$ & $12 \pm 5$ \\
HoAcAc nanoparticles & $187 \pm 4$ & $198 \pm 3$ & $14 \pm 7$ \\
\hline
\end{tabular}

where field inhomogeneities caused by holmium are dephased before molecular diffusion can average out this signal. In nanoparticle dispersions, however, molecular diffusion of protons averages out the local field inhomogeneities, thereby reducing the $\mathrm{r}_{2}{ }^{*}$ of HoAcAc nanoparticles compared to HoAcAc microspheres $(21,22)$.

An MRI phantom setup was used to determine the minimum detectable amount of HoAcAc nanoparticles at 3 Tesla using a typical $\mathrm{T}_{2}$ * weighted scan technique (Fig. 5). The observed minimum detectable amount was $1.5 \mu \mathrm{g}$ and equals that of HoAcAc microspheres (9), which is explained by the deposits in which the HoAcAc nanoparticles are packed together in the phantom mimicking larger particles $(21,23)$.

Holmium is a more potent CT contrast agent than the routinely used iodine-based agents (6). The effect of holmium on CT is related to its mass attenuation coefficient $\mu$. The $\mu$ value was determined by constructing a linear curve as described by Seevinck et al. (6). The $\mu$ value is $16 \mathrm{HU} \mathrm{mg}^{-1}$ for the HoAcAc nanoparticles, which is similar to that of HoAcAc microspheres (9). This was expected, since the $\mu$ value is not dependent on the particle size, but only dependent on the holmium concentration in the phantom (6).

Neither significant changes in the size distribution nor in particle surface were observed after neutron irradiation of $50 \mathrm{mg}$ HoAcAc nanoparticles ( $1 \mathrm{~h}$ irradiation time and a neutron flux of $5 \times 10^{12} \mathrm{n} \mathrm{cm}^{-2} \mathrm{~s}^{-1}$ (Fig. 1c)) and resulted in
$600 \mathrm{MBq}$ of holmium-166. This activity is sufficient for treatment of a single, solitary, inoperable tumor $(<3 \mathrm{~cm})$ by intratumoral injection (9). Additionally, the gamma rays emitted by holmium-166 can be visualized by gamma scintigraphy, the results of which can be used for tumorabsorbed dose quantification (24).

A limiting factor in intratumoral injection of a microsphere suspension is the aggregation and settling of the microspheres in the syringe prior to administration. In a renal cell carcinoma mouse model, this resulted in the administration of only half of the intended dose of HoAcAc microspheres (10). According to Stokes' law, the sedimentation velocity of a particle in a dispersion medium is proportional to $\mathrm{d}^{2}$, which is the particle diameter in centimeter (25). The HoAcAc nanoparticles described in the present study have an approximately 200-fold lower diameter compared to the HoAcAc microspheres, which reduces the sedimentation velocity by a factor 40,000. Also, the positive zeta potential of the HoAcAc nanoparticles contributes to prevent aggregation. In this way, a suspension of HoAcAc nanoparticles prepared in DMAB can be obtained, which seems ideally suited for intratumoral injections. In large tumors, multisite injections of microsized holmium particles are required to obtain a homogeneous tumor irradiation. In tumors in or near delicate structures, only single injections can be administered. One might even speculate that the use of nano-sized particles results in a homogeneous particle distribution throughout the tumor, following a single injection, but this is subject of further investigation.

\section{CONCLUSION}

In conclusion, the present study demonstrates that HoAcAc nanoparticles can be prepared using a solvent evaporation process. The particle diameter can easily be adapted and a

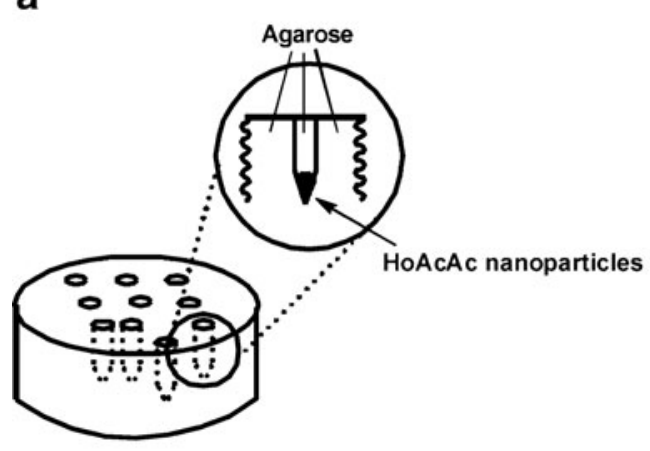

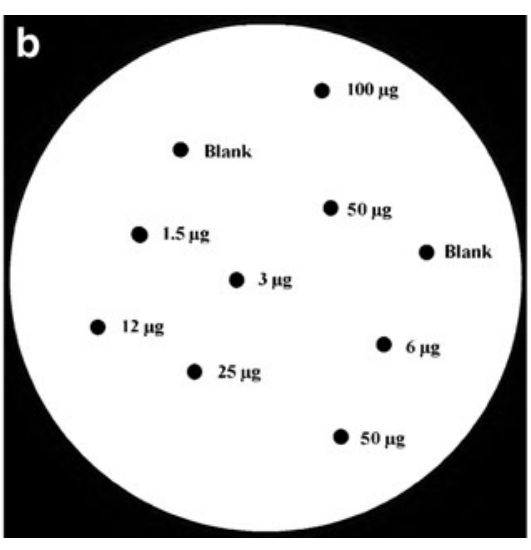

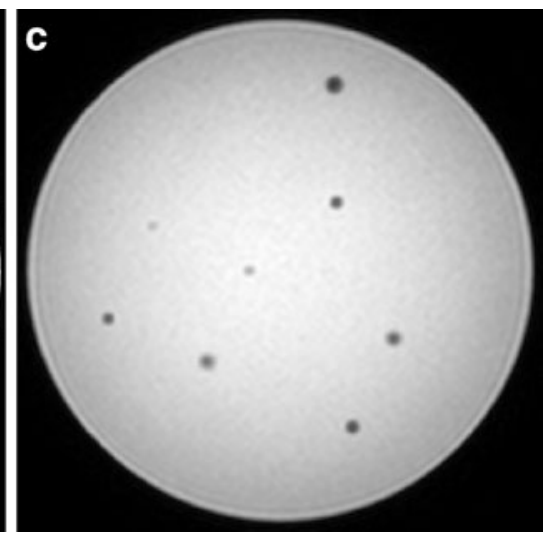

Fig. 5 Agarose MRI phantom setup for the determination of the minimally visible amount of nanoparticles. a) shows how the phantom was constructed, b) shows a schematic representation of the phantom layout and the amounts of nanoparticles in $\mu \mathrm{g}$, $\mathbf{c}$ ) shows the actual image at $3 \mathrm{~T}$. 
can be optimized for specific therapeutic applications and tumor types. The element holmium is very suitable as a contrast agent, since it is one of the only true multimodality (radio)nuclides, allowing for MR imaging, CT imaging and gamma scintigraphy. Moreover, holmium can be made sufficiently radioactive to exert a therapeutic effect when injected intratumorally.

\section{ACKNOWLEDGEMENTS}

Financial support by the Dutch Technology Foundation under grant 06069 is gratefully acknowledged. The authors would like to thank miss X.H. Lie for help with preparing the particles, Dr. W.H. Müller, Mr. G.J.M.M. Schneijdenberg and Mr. J.D. Meeldijk for their assistance with SEM image acquisition. The valuable discussions with Dr. T. Visser and Dr. M. van de Weert are gratefully acknowledged. Ms. M.J.J. Koster-Ammerlaan is acknowledged for her help with the neutron irradiation experiments.

Open Access This article is distributed under the terms of the Creative Commons Attribution Noncommercial License which permits any noncommercial use, distribution, and reproduction in any medium, provided the original author(s) and source are credited.

\section{REFERENCES}

1. Jemal A, Siegel R, Ward E, Hao Y, Xu J, Thun MJ. Cancer statistics, 2009. CA Cancer J Clin. 2009;59:225-49.

2. Kennedy JE. High-intensity focused ultrasound in the treatment of solid tumours. Nat Rev Cancer. 2005;5:321-7.

3. Liapi E, Geschwind JF. Transcatheter and ablative therapeutic approaches for solid malignancies. J Clin Oncol. 2007;25:978-86.

4. Tian JH, Xu BX, Zhang JM, Dong BW, Liang P, Wang XD. Ultrasound-guided internal radiotherapy using yttrium-90-glass microspheres for liver malignancies. J Nucl Med. 1996;37:958-63.

5. Firusian N, Dempke W. An early phase II study of intratumoral P32 chromic phosphate injection therapy for patients with refractory solid tumors and solitary metastases. Cancer. 1999;85:980-7.

6. Seevinck PR, Seppenwoolde JH, de Wit TC, Nijsen JF, Beekman FJ, van het Schip $\mathrm{AD}$, et al. Factors affecting the sensitivity and detection limits of MRI, CT, and SPECT for multimodal diagnostic and therapeutic agents. Anticancer Agents Med Chem. 2007;7:317-34.

7. Lee JD, Yang WI, Lee MG, Ryu YH, Park JH, Shin KH, et al. Effective local control of malignant melanoma by intratumoural injection of a beta-emitting radionuclide. Eur J Nucl Med Mol Imaging. 2002;29:221-30.

8. KimJK, Han KH, Lee JT, Paik YH, Ahn SH, Lee JD, et al. Long-term clinical outcome of phase IIb clinical trial of percutaneous injection with holmium-166/chitosan complex (Milican) for the treatment of small hepatocellular carcinoma. Clin Cancer Res. 2006;12:543-8.
9. Bult W, Seevinck PR, Krijger GC, Visser T, Kroon-Batenburg LM, Bakker CJ, et al. Microspheres with ultrahigh holmium content for radioablation of malignancies. Pharm Res. 2009;26:1371-8.

10. Bult W, Kroeze SGC, Elschot M, Seevinck PR, Beekman FJ, Luijten PR, et al. Intratumoral administration of holmium-166 acetylacetonate microspheres as a novel minimally-invasive treatment for small kidney tumors. Cancer Res. 2010; submitted.

11. Nijsen JFW, Zonnenberg BA, Woittiez JR, Rook DW, Swildensvan Woudenberg IA, van Rijk PP, et al. Holmium-166 poly lactic acid microspheres applicable for intra-arterial radionuclide therapy of hepatic malignancies: effects of preparation and neutron activation techniques. Eur J Nucl Med. 1999;26:699-704.

12. Vente MA, Nijsen JF, de Roos R, van Steenbergen MJ, Kaaijk CN, Koster-Ammerlaan MJ, et al. Neutron activation of holmium poly (L-lactic acid) microspheres for hepatic arterial radio-embolization: a validation study. Biomed Microdevices. 2009;11:763-72.

13. Filipe V, Hawe A, Jiskoot W. Critical Evaluation of Nanoparticle Tracking Analysis (NTA) by NanoSight for the measurement of nanoparticles and protein aggregates. Pharm Res. 2010;27:796-810.

14. Kwon HY, Lee JY, Choi SW, Jang YS, Kim JH. Preparation of PLGA nanoparticles containing estrogen by emulsificationdiffusion method. Colloids Surf, A. 2001;182:123-30.

15. Nijsen JFW, van Steenbergen MJ, Kooijman H, Talsma H, Kroon-Batenburg LM, van de Weert M, et al. Characterization of poly(L-lactic acid) microspheres loaded with holmium acetylacetonate. Biomaterials. 2001;22:3073-81.

16. Kooijman H, Nijsen F, Spek AL, van het Schip AD. Diaquatris (pentane-2, 4-dionato-O, $\mathrm{O}^{\prime}$ )holmium(III) monohydrate and diaquatris(pentane-2, 4-dionato-O, O')holmium(III) 4-hydroxypentan2-one solvate dihydrate. Acta Crystallogr C. 2000;56:156-8.

17. Dillen AJ, Terode RJAM, Lensveld DJ, Geus JW, de Jong KP. Synthesis of supported catalysts by impregnation and drying using aqueous chelated metal complexes. J Catal. 2003;216:25764.

18. Feng S, Huang G. Effects of emulsifiers on the controlled release of paclitaxel (Taxol) from nanospheres of biodegradable polymers. J Control Release. 2001;71:53-69.

19. Seppenwoolde JH, Nijsen JFW, Bartels LW, Zielhuis SW, van het Schip AD, Bakker CJ. Internal radiation therapy of liver tumors: qualitative and quantitative magnetic resonance imaging of the biodistribution of holmium-loaded microspheres in animal models. Magn Reson Med. 2004;53:76-84.

20. Fossheim S, Johansson C, Fahlvik AK, Grace D, Klaveness J. Lanthanide-based susceptibility contrast agents: assessment of the magnetic properties. Magn Reson Med. 1996;35:201-6.

21. Seevinck PR, Seppenwoolde JH, Zwanenburg JJM, Nijsen JFW, Bakker CJG. FID sampling superior to spin-echo sampling for T$2 *$-based quantification of holmium-loaded microspheres: theory and experiment. Magn Reson Med. 2008;60:1466-76.

22. Yung KT. Empirical models of transverse relaxation for spherical magnetic perturbers. Magn Reson Imaging. 2003;21:451-63.

23. Kuhlpeter R, Dahnke H, Matuszewski L, Persigehl T, von Wallbrunn A, Allkemper T, et al. R2 and R2* mapping for sensing cell-bound superparamagnetic nanoparticles: in vitro and murine in vivo testing. Radiology. 2007;245:449-57.

24. de Wit TC, Xiao J, Nijsen JF, van het Schip FD, Staelens SG, van Rijk PP, et al. Hybrid scatter correction applied to quantitative holmium-166 SPECT. Phys Med Biol. 2006;51:4773-87.

25. Martin AN. Coarse dispersions. In: Martin AN, editor. Physical pharmacy. Philadelphia: Lea \& Febiger; 1993. p. 477-511. 